\title{
Enhancing Performance of TCP Variants in LTE
}

\author{
Saleh M. Abdullah \\ Faculty of Computers \\ and Information, \\ Menoufia University , \\ Egypt
}

\author{
Osama Younes, PhD \\ Faculty of Computers \\ and Information \\ Menoufia University, \\ Egypt
}

\author{
Hamdy M. Mousa \\ Faculty of Computers \\ and Information , \\ Menoufia \\ University, Egypt
}

\author{
Hatem Abdul-kader \\ Faculty of Computers \\ and Information , \\ Menoufia \\ University, Egypt
}

\begin{abstract}
Long Term Evolution (LTE) is the 3rd Generation Partnership Project (3GPP), all-IP wireless protocol that evolved from Global System for Mobile Communications (GSM). Congestion control algorithms are an important part of Transmission Control Protocol (TCP) that directly determines the performance of the protocol in the IP Network. Several TCP variants have been proposed. It can be used to enhance quality of service (QoS). In this paper, an enhanced approach is proposed to improve the performance of LTE network. The proposed approach depends on controlling the receive buffer size in eNode B. This will be performed through dividing the memory size of eNode B between active users. Simulation results show the effectiveness of the proposed approach that lead to enhance performance in terms of network throughput and low time delay.
\end{abstract}

\section{Keywords}

Congestion control ; dynamic window; throughput; LTE; TCP; OPNET.

\section{INTRODUCTION}

LTE is $4^{\text {th }}$ Generation wireless broadband technology developed by $3 \mathrm{GPP}$. By compared it with the earlier wireless systems, LTE increases the capacity, coverage and speed and it also provides a spectrum and significant increasing in spectrum efficiency [1]. Furthermore, it takes into account different architecture in radio network that's designed to speed up the operations and to decrease the cost. Since the end of 2009, the LTE has been installed as a normal growth of GSM and Universal Mobile Telecommunications System (UMTS) [2].

LTE uses access techniques Frequency Division Duplexing (FDD) and Time Division Duplexing (TDD) [3]. LTE has focused on data services that supports only the Packet Switched (PS) domain, Packet-switched networks use buffers to accommodate transient traffic bursts. The functionality of these buffers is prevent packet loss and maintain high output link utilization. Packet loss may happen in the buffer of a node, if the size of the buffer becomes less than the flow of packets into the buffer, for overcoming the loss of packets in the buffer, it can be reduced by setting the threshold limit and if it is reached then the rate of flow of packets should be reduced. The threshold set for the buffer is the buffer size [4]. One of the most essential request of $4 \mathrm{G}$ systems is that the users should not feel any difference between a wired and a wireless network and they should have multiple options for connectivity over heterogeneous networks. TCP protocol is able to achieve these requirements. The immediate approach to enhance the performance of TCP for the new generation. networks is to modify the TCP itself, due to TCP still represents one of the major reasons of poor performance in a wireless environment [5]. The static window size technique appeared usually have more data in flight, where the fight is
Amount of data that has been sent but not yet acknowledged (acked).however, the sender has no way to estimate the capacity of the link other than to fill the network and its queues and induce packet loss. Static window works as well if the bottleneck service rate along the path and round trip time are fixed. Wherever, bottleneck rate changes with time. Static choice of flow control window can lead to problems such as too small or too large, its need to adapt window, always try to get to the current optimal value. To overcome this problem, the sending rate should be adapted as a function of the available capacity of the eNode $B$ rate.

In this paper, an approach is proposed to avoid congestion in the buffer by controlling the receive buffer size of TCP in eNode B. The proposed approach depends on Adaptive configurations of window size according to the LTE traffic. The congestion mechanisms make a dynamic change of window size of TCP flow according to the maximum buffer size and the number of users in the cell.

The paper is organized as the following. The LTE network architecture is presented in Section 2. Section 2 discusses the simulator model setup. Section 3. is presented related work. Section 4 discusses the proposed approach. Performance evaluates and analyzes in section 5. Finally, the conclusion is given in Section 6.

\section{BACKGROUND}

LTE network technology will be used for fixed, portable and mobile wireless broadband access. It will offer several benefits to operators. It also aims to decrease network complexity, increasing capacity, lowering deployment and operational costs. It will enable operators to facing up the growing demands for mobile data solutions and making it possible for richer services to be delivered to consumers more cost effectively [6].

LTE Advanced (LTE-A) is evolved on the regular LTE standard, that aiming to improve network capacity and increase transmission rate for consumers. The most important thing to know is that LTE-A promising to achive true $4 \mathrm{G}$ speeds, unlike current LTE networks. You can expect the realworld speed of LTE-A to be more two times faster than today's LTE. It should also be strong, with fewer dropped connections as you move around [7].

\subsection{LTE Network Archtecture}

The architecture describes the main functional components of LTE network as shown in Fig.1 [8]. Core Network and Access Network, which consider an IP layer of LTE System. The non-radio component is System Architecture Evolution (SAE) that is comprised of Evolved Packet Core (EPC) network. SAE and LTE are the Evolved Packet System (EPS). 


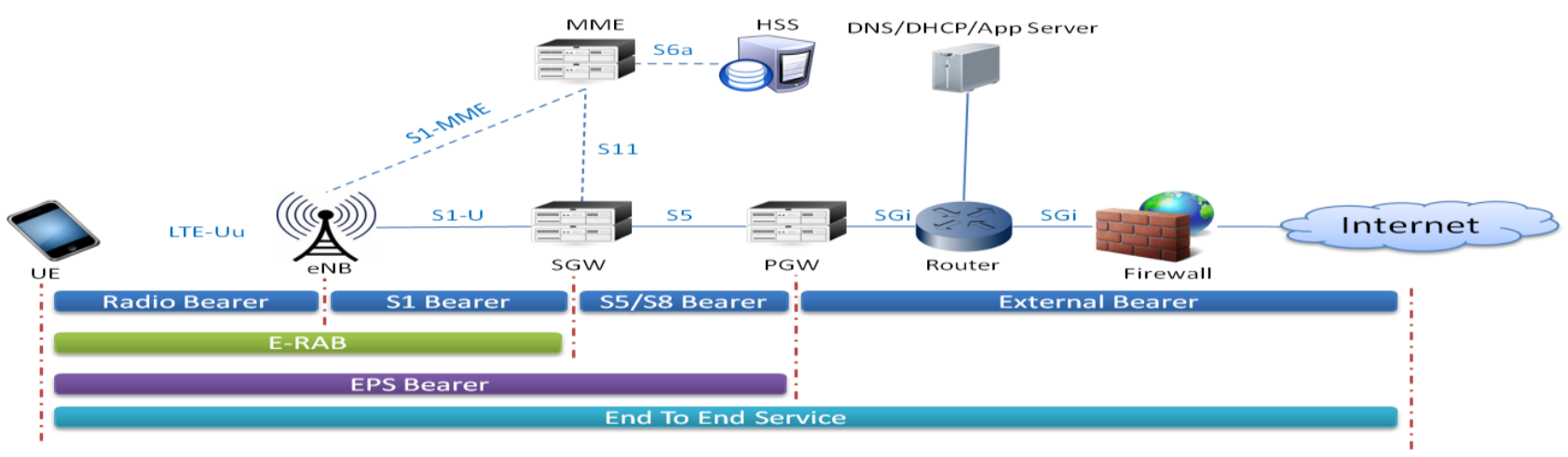

Fig 1. LTE reference architecture [8]

The eNode B is one of the key components of the E-UTRAN and connects the transport network with the users' side. The eNode B contains all the radio interface related protocols such as Physical layer, Media Access Control(MAC) Layer, Radio Link Control (RLC) and Packet Data Convergence Protocol ( PDCP). It also contains the transport protocols such as a General Packet Radio Service (GPRS) Tunneling Protocol (GTP), User Datagram Protocol (UDP) and IP. The Home Subscriber Server (HSS) is a central data base that contains on all information about the network operator's subscribers. The Packet Data Network (PDN) Gateway (P-GW) connects with the out world. It do the same function of the (GPRS) support node (GGSN) and the serving (GPRS) support node (SGSN) that are used in UMTS and GSM. The serving gateway (S-GW) is a router and its function forwards data between the base station and the PDN gateway. The The Mobility Management Entity (MME) is control-node that responsible for idle mode User Equipment (UE) paging and tagging procedure including retransmissions. The interfaces of the system can be explained as follows [8-9]:

- The S1-U interface uses GTP-U on UDP/IP transport and provides non-guaranteed delivery of user plane PDUs between theeNode B and the SGW.

- The S1 control plane interface (S1-MME) uses between eNode B and MME.

- $\quad$ S5 provides managed for user plane tunneling and tunnel between Serving GW and PDN GW.

- $\quad$ S8 is an Inter-PublicLand Mobile Network (PLMN) reference point providing user and control plane between the Serving GW in the VPLMN (Visited PLMN) and the PDN GW in the HPLMN (Home PLMN).

- The s11 interface uses control plane between MME and SGW. It provides GTP tunnel per user.

- S6a interface utilizes between MME and HSS. It transfers data of authenticating/authorizing user access to the evolved system

- SGi interface is the reference point between the PDN GW and the packet data network.

- MME is used for overall control of the user equipment (UE).

\subsection{LTE Opnet Simulator Model}

The proposed LTE model consists of a number of nodes and entities such as UE node, eNode B, aGW, FTP and HTTP OPNET application/profile, default QoS configuration,
Mobility configuration node, FTP and HTTP OPNET remote server and global information center node in OPNET simulator as shown in Fig. 2 This model is tested with FTP and HTTP services.

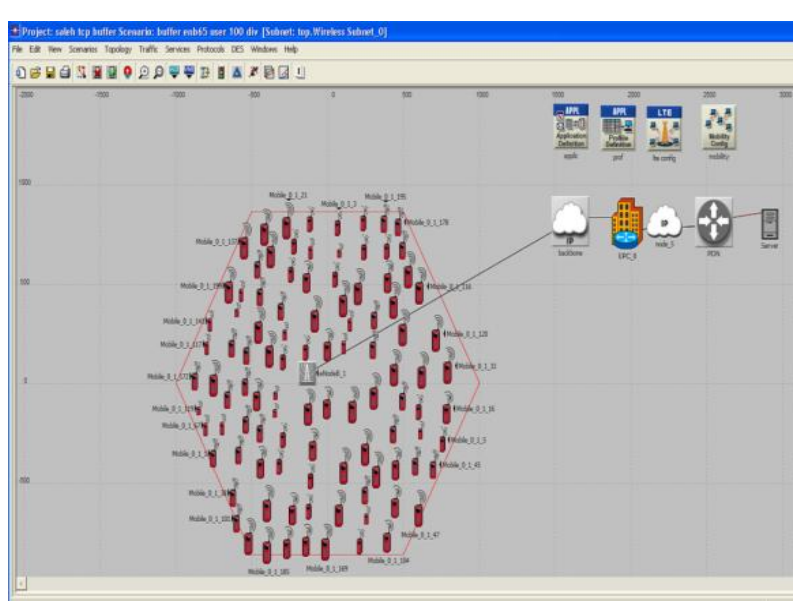

Fig 2. LTE Network model

\subsection{TCP Protocol}

$\mathrm{TCP} / \mathrm{IP}$ is the standard networking protocol stack for Internet. TCP is a connection oriented protocol working at transport layer to control communication between links. So it is expected to be deployed over wireless networks to allow seamless integration with the Internet. However, earlier research suggested that TCP performs poorly over cellular (single-hop) wireless networks [10]. There are various types of TCP variants which are developed to control congestion in network with maintaining high throughput in the network [11]. The following TCP flavors are used for analysis of traffic in LTE network.

TCP-Tahoe utilizes the slow-start (SS), congestion avoidance (CA) and fast retransmit algorithms (RFC 2001).Congestion avoidance in TCP Tahoe relies on setting the cwnd to half the current window size on timeout. afterward, on each ACK for new data, the cwnd is increased by $1 /$ cwnd.

TCP-Reno define congestion window to one packet upon a time out retransmission timeout (RTO). Reno extended its algorithm to include the fast retransmit mechanism. In the fast retransmit mechanism, when three duplicate ACKs for a packet are received before the RTO, the dropped packet is retransmitted. It is also to prevent transmission to re-enter the slow start state after a fast retransmit, Fast recovery mechanisms are introduced in Reno. Instead, the window size is halved and the threshold is adjusted accordingly and TCP remains in congestion avoidance until a timeout occurs. TCP 
Reno became the standard TCP algorithm implemented in most computers. Reno algorithms are a slow start, congestion avoidance, fast retransmit and fast recovery [12].

TCP- New Reno uses two variables, the congestion window size (cwnd), which initially set to one segment, and slow-star (SS) Threshold (ssthresh). At the beginning of the TCP connection, the sender enters the Slow Start (SS) phase. For every ACK receiving, the cwnd increases by one segment. When reaching the ssthresh of cwnd, the Congestion Avoidance (CA) phase starts and increases the cwnd by $1 /$ cwnd for every receiving ACK for slowly probe the network bandwidth availability. If cwnd reaches the receiver's advertised window, or by the reception of 3 DUPACKs, this linear growth ends. In the last case, TCP infers that lost packets due to link congestion and it decrease the current value of cwnd by half of its value attempting to prevent network collapse (Fast Recovery). This is Additive Increase Multiplicative Decrease (AIMD). TCP activates congestion control, prevents avoidance mechanism and incapable of handling efficiently mixed-type packet losses happening in wired/wireless heterogeneous networks.

TCP-Cubic algorithm is optimized congestion control that adjusts cwnd with different way. It utilizes cubic function instead of a linear window increase of the current TCP standards to improve scalability and stability under fast and long distance networks for high bandwidth networks with high latency $[13,14]$.

TCP -Standard no utilizes Fast recovery algorithms of packets, flows with very short round time trip (RTTs) will receive ACKs faster and therefore have their congestion windows grow to go faster than other flows with longer RTT.

\section{RELATED WORKS}

Liang Zhao at al. [15] described the Long term Evolution (LTE) Network that is the next-generation network technology beyond 3G. When dealing with the traffic bursts in wireless due to environmental interferences, buffer overflow occurs often in eNode B of the LTE network. To prevent the buffer from overflow, the advertisement window is approximately controlled in order to enhance TCP-sending. The data rate will be slowed down by the sender upon the receipt of the reduced advertisement window, and hence the buffer overflow becomes alleviated. The result performance in terms of network throughput enhanced, average packet delay and jitter, as well as rate of packet loss reduced. However, the weaknesses of this technique that don't support handover in the LTE network and congestion coefficient and the adjustment coefficient of the mechanism are configured with fixed values.

In [16], schedulers allocate resources without taking congestion at the (eNode B's) output buffer into calculation. This Lead for buffer overflows and damage in overall QoS. Congestion avoidance and fair bandwidth allocation is considered in existent research for the LTE and LTEAdvanced uplink connections. This article provide a technique for LTE and LTE-Advanced, LTE Fair Intelligent Congestion Control (LTE_FICC), to control congestion at an eNode B. LTE_FICC overcome the problem of unfair bandwidth allocation among the flows which share the same eNode B interface. The results show that LTE_FICC controls the eNode B buffer effectively prevents overflows; and guarantee the QoS of flows in terms of fair bandwidth allocation, enhance throughput and reduced queuing delay. However, setting the target point fixed, at a level that is not suitable for the network performance and this technique not an effective admission control algorithm that works together with the congestion control scheme to minimize the end to end delay of the network connections.

A buffer overflow notification protocol is proposed in $[17,18]$ to control the overflow of buffer effectively at the link layer in the wireless ad hoc networks. The higher transit priority is allowed to the congested node by the protocol so as to access a shared medium by the dynamic adjustment of the contention window of the node at its neighboring node. Simulation results show that $10-20 \%$ enhance of throughput is achieved by the buffer overflow notification protocol. The packet loss becuase buffer overflow is immediately and efficiently reduced in intermediate nodes on the transmission path. Nevertheless, this methodology is not to determine the optimal cumulative neighborhood buffer in multi-flow environments and the per-flow buffer (of 1-3 packets) at each relay node is still small.

\section{PROPOSED APPROACH}

Generally, eNode B will not interpret the layers above Packet

Data Convergence Protocol (PDCP).

The eNode B buffer congestion can be identified by the number of buffered packets exceeds the predefined congestion threshold, cth, which is determined by

$$
\text { cth }=b_{s} * \alpha ; 0<\alpha \leq 1
$$

Where $b_{s}$ and $\propto$ are the size of eNode $\mathrm{B}$ buffer and a congestion coefficient respectively. RLC in the eNode B monitors its buffer space utilization once a packet enters or leaves the queue.

In the LTE network, buffer overflow in the eNode B can occur under either of the following two conditions: (1) the incoming traffic data rate exceeds the bandwidth of the air interface. The RLC packets will be buffered in the eNode B and be dropped when the buffer overflows; and (2) various environmental interferences can cause RLC packet loss during transmission in the wireless part of the network. Thus, the data sending rate needs to be well controlled so as to avoid the eNode B's buffer overflow whilst minimize the system performance degradation.

In this paper, dynamic window control technique is proposed which adoption congestion window size based on the available capacity of eNode B. When the connection between the user and the eNode B is established, the window control technique will be allocated to each user buffer through the following formula based on the size of the buffer in the eNode B:

$$
B_{u}=\frac{\mathrm{B}_{\text {eNode B }}}{\mathrm{N}}
$$

Where $\mathrm{B}_{\text {eNode }} \mathrm{B}$ is the size of eNode $\mathrm{B}$ buffer, $B_{u}$ the size of user buffer and $\mathrm{N}$ number of user active.

When the user incoming or out coming in eNode B, The eNode B advertises how much space it has available in its buffers. The window control technique will reallocate buffer size for each user, and the users are restricted its transmission data rate (Receiver's advertised window (rwnd)) so that it will reduce congestion in eNode $\mathrm{B}$.

To avoid a fast sender from flood a slow receiver with more segments than its buffer capacity, TCP has been designed to provide the receiver with full control of the flow rate. The window field in the TCP header is used to implement a sliding window scheme to achieve this. Every ACK sent by the receiver contains a value in the window field which reflects the available buffer capacity at its end (this value could also 
be zero, if the buffer is full). The sender steps its transmission rate based on this value.

One of the most important attribute for enhancing the connection establishment is the Maximum Segment Size (MSS) that defines the largest amount of data that will be transmitted as one TCP segment. Choosing a good MSS value is important because if MSS is small, network utilization may be low; if MSS is large, it degrades performance. In dynamic case, this attribute is setting to "Auto-Assigned" for both the server and the eNode B to automatically guarantee optimal TCP operating. While this attribute is setting to 512 byte at user. In static case, this attribute is setting to "Auto-Assigned" for user, eNode B and server.

Another important attribute is TCP Window Scaling that must be set to "Enabled" at the UE, while at the server host, it set to either "Enabled" or "Passive". If the UE has the attribute set to "Passive", Window Scaling is disabled at the server host.

\section{PERFORMANCE EVALUATIO AND ANALYSIS}

The performance assessment of the proposed approach describes in this section and it presents a set of key results. The Key parameters of simulation network are listed in Table I.

TableI: Simulation Configuration Parameter

\begin{tabular}{|c|c|c|c|}
\hline TCP Parameter & Users & eNode B & server \\
\hline Initial RTO & $3.0 \mathrm{~s}$ & $3.0 \mathrm{~s}$ & $3.0 \mathrm{~s}$ \\
\hline $\begin{array}{c}\text { maximum Sender } \\
\text { segment size } \\
\text { (SMSS) }\end{array}$ & 512(byte) & Auto-assigned & $\begin{array}{c}\text { Auto- } \\
\text { assigned }\end{array}$ \\
\hline $\begin{array}{c}\text { Slow start initial } \\
\text { count }\end{array}$ & 2 & 2 & 2 \\
\hline $\begin{array}{l}\text { Retransmission } \\
\text { threshold }\end{array}$ & $\begin{array}{l}\text { Attempts } \\
\text { based }\end{array}$ & Attempts based & $\begin{array}{c}\text { Attempts } \\
\text { based }\end{array}$ \\
\hline RTT gain & 0.125 & 0.125 & 0.125 \\
\hline $\begin{array}{c}\text { Duplicate ACK } \\
\text { threshold }\end{array}$ & 3 & 3 & 3 \\
\hline $\begin{array}{c}\text { RTT deviation } \\
\text { coefficient }\end{array}$ & 4 & 4 & 4 \\
\hline $\begin{array}{l}\text { RTT deviation } \\
\text { gain }\end{array}$ & 0.25 & 0.25 & 0.25 \\
\hline $\begin{array}{l}\text { Maximum ACK } \\
\text { segment }\end{array}$ & 2 & 2 & 2 \\
\hline $\begin{array}{c}\text { Maximum ACK } \\
\text { delay }\end{array}$ & $0.200 \mathrm{~s}$ & $0.200 \mathrm{~s}$ & $0.200 \mathrm{~s}$ \\
\hline Persistent timeout & $1.0 \mathrm{~s}$ & $1.0 \mathrm{~s}$ & $1.0 \mathrm{~s}$ \\
\hline Timer granularity & $0.5 \mathrm{~s}$ & $0.5 \mathrm{~s}$ & $0.5 \mathrm{~s}$ \\
\hline Maximum RTO & $64.0 \mathrm{~s}$ & $64.0 \mathrm{~s}$ & $64.0 \mathrm{~s}$ \\
\hline Minimum RTO & $1.0 \mathrm{~s}$ & $1.0 \mathrm{~s}$ & $1.0 \mathrm{~s}$ \\
\hline Fast recovery & Enable & Enable & Enable \\
\hline Fast retranslation & Enable & Enable & Enable \\
\hline Window scaling & Enable & Enable & Enable \\
\hline $\begin{array}{c}\text { Receive buffer } \\
\text { adjustment }\end{array}$ & $\begin{array}{c}\text { Window } \\
\text { based }\end{array}$ & Window based & none \\
\hline
\end{tabular}

\subsection{Throughput Performance}

The total amount of data packet, which is successfully delivered to destination node from source node over communication network is called throughput. The throughput is measured in bits per second (bits/sec). Mathematically, throughput can be defined by the following formula [19]. throughput $=\frac{\text { number of delivered packet } * \text { packet size }(\text { Bytes }) * \text { size }}{\text { total duration of simulation }(\sec )}$ (3)

To study the effect of number of users in the throughput for all types of TCP with both cases; dynamic and static. The experimental is setup in simulator. The experimental results is shown in Figures s 3 and 4 that illustrate throughput in both cases; with varying the number of users. It is noted that there are great similarities in all types except in some cases which will be describe later. When the number of users is 10 , it is noted that the throughput is less than the case of 25 or 50 users. This is due to the buffer size allocated to each user in this case is large. So it needs to be high-delay Then happen lost packets this leads to not fully delivered packets, compared to when you have the number of users more; the buffer size allocated for each user is less; so that can complete the data transfer.

In Fig.5 It is noted that high throughput in TCP New Reno and cubic, and less in the other variant. When the number of users is few then the window size for each user be large and thus need high delay, high packet loss and throughput is low. In TCP-New Reno biggest percentage achieved because it uses a mechanism fast recovery in case of loss of multipackets, sense astray repeated use fast recovery in the same window, thus reducing the rate of lost packets and improve the performance of throughput.

TCP-Reno waits for a retransmit time-out whenever multiple packets are lost from the same transmission window, thus, can't make the sender to continue in fast recovery until all the packets which were outstanding during the start of the fast recovery have been acknowledged. Entering into fast recovery multiple times. This causes high delay and lose a lot of data and thus data is delivered less than.

TCP-Thao and TCP-Standard no utilizes Fast recovery algorithms of packets. They are switching to slow start instead of congestion avoidance phase. This behavior no improves the network throughput.

TCP-cubic achieves more equitable bandwidth allocations among flows with different RTTs (round trip times) by making the window growth to be independent of RTT - thus those flows grow their congestion window at the same rate. This feature allows CUBIC to be very scalable when the bandwidth and delay product of the network is large, and at the same time, be highly stable and also fair to standard TCP flows. This behavior improves the network throughput.

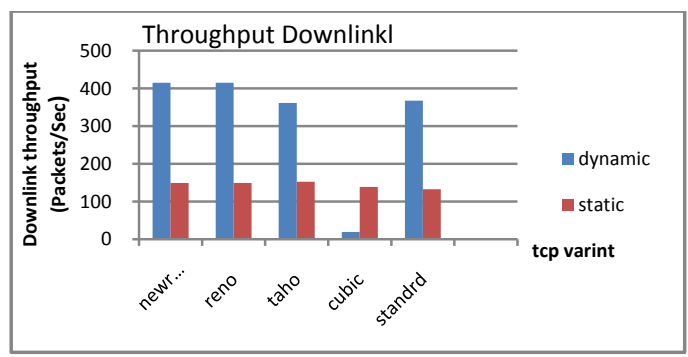

Fig 3. Throughput for each TCP with users 50 


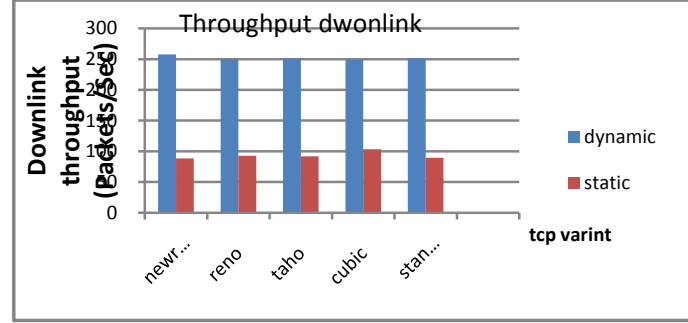

Fig 4. Throughput for each TCP with users 25

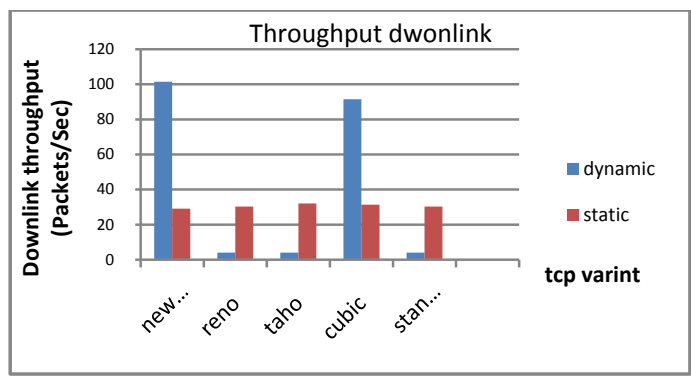

Fig 5. Throughput for each TCP with users 10

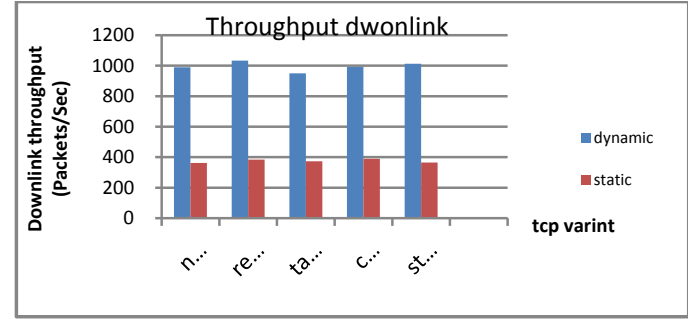

Fig 6. Throughput for each TCP with users 100

In the following Figures from 7 to 10 , it is noted that the TCP-Reno has the biggest percentage throughput that excesses 1000 packets compared to other variant as result of the client switches to the fast recovery phase after fast retransmit in TCP-Reno, when it receives 3 duplicate acknowledgements instead of switching to slow-start as Tahoe. The reason for going to fast recovery instead of slow start is to allow the sender to work in congestion avoidance phase. This behavior improves the network throughput as more packets will be transmitted.

The throughput with each type of TCP is shown in Figures from 7 to 10 . The greatest value is achieved with 100 users, because of the small size of the buffer for each user. While fewer throughputs is achieved with 10 users due to large buffer size allocated for each user.

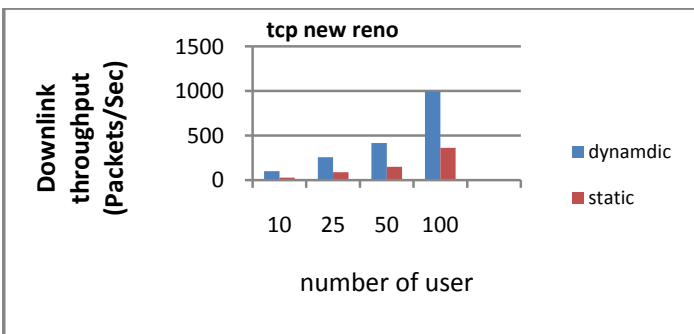

Fig 7. Throughput withTCP New Reno

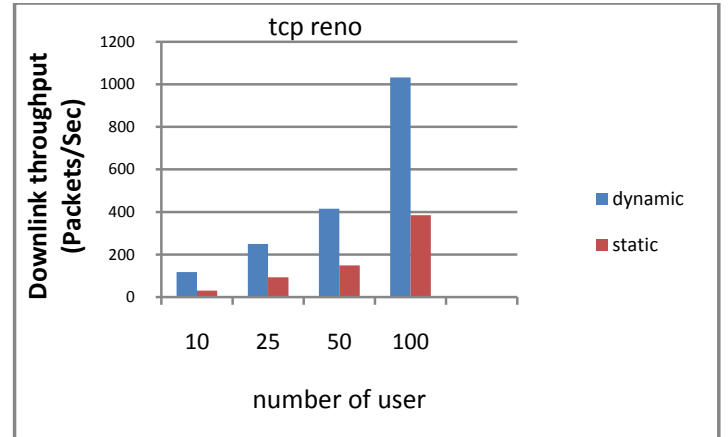

Fig 8. Throughput withTCP Reno

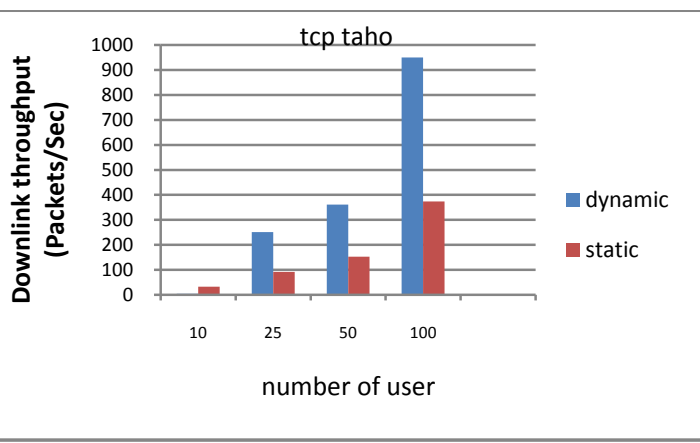

Fig 9. Throughput with TCP Thao

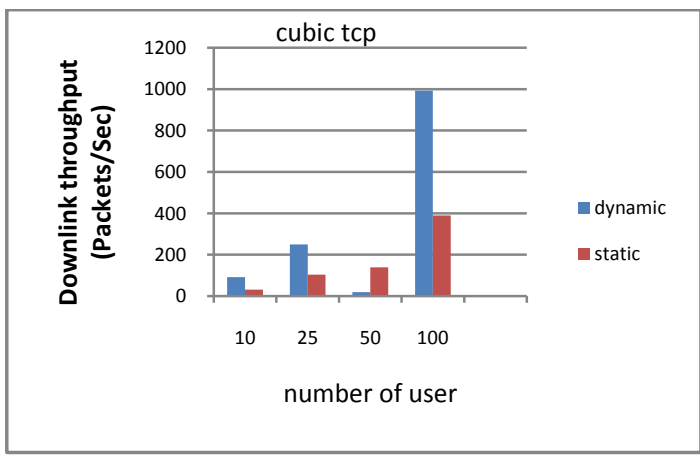

Fig 10. Throughput with TCP cubic

\subsection{LTE Global Parameters (Delay)}

LTE delay is the round-trip delay, which is the time from a packet is send to the acknowledgement is received. This delay is related to the distance between the user and base station.

To study the effect of number of users in the delay in both cases dynamic and static for all types of TCP, the experimental results are shown in the following Figures from 11 to 14 that illustrate delay in both cases. That has been evaluated and improved performance. When a various number of users, there are great similarities in all types except in some cases which will be explained in the following. With 10 users, the results show that the delay is larger than in the case with 25 or 50 users. This is due to be allocated to each user buffer size when the number of 10 is bigger than the rest of cases, and thus it causes high-delay. 


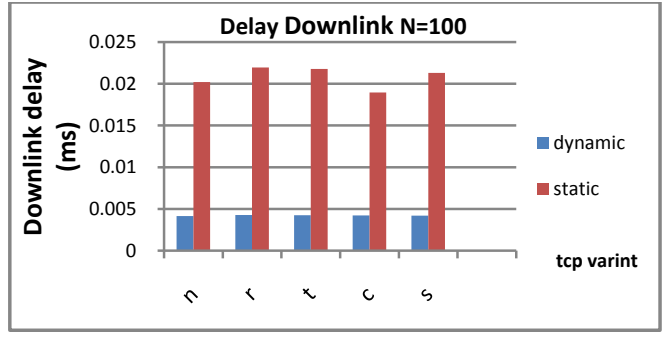

Fig 11. Delay for each TCP with users 100

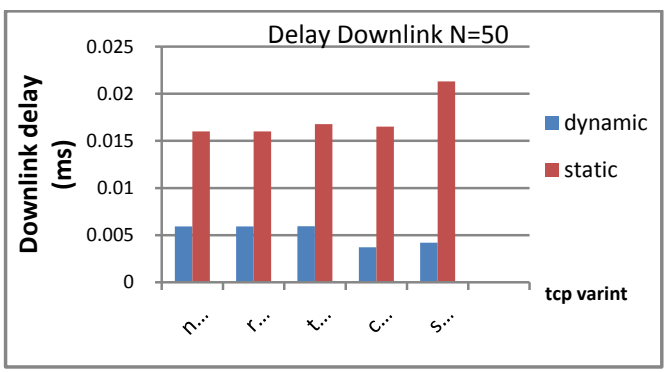

Fig 12. Delay for each TCP with users 50

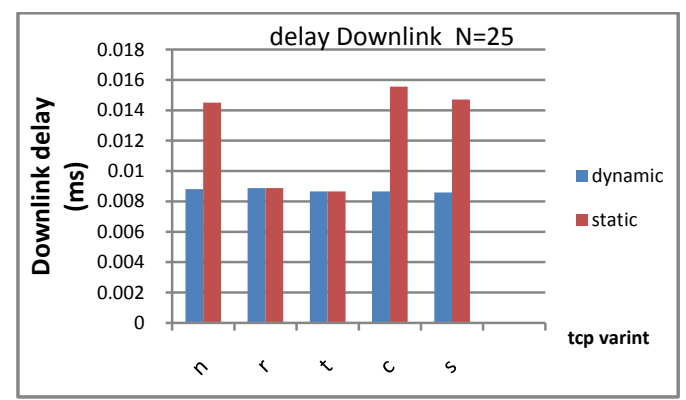

Fig 13. Delay for each TCP with users 25

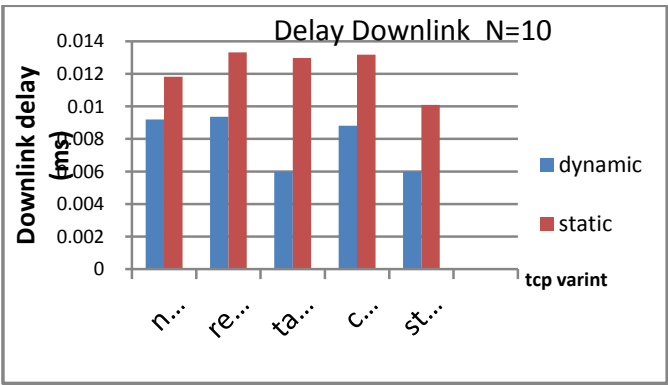

Fig 14. Delay for each TCP with users 10

The following Figures from 15 to18 show the delay with each type TCP. The greatest value achieves when the number of users 10, Because of the large size of the buffer for each user, while less delay value is getting with 100 users due to small buffer size allocated for each user.

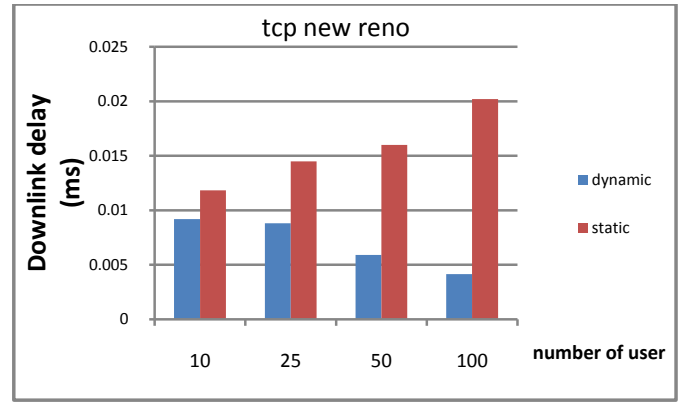

Fige 15. Delay with New Reno

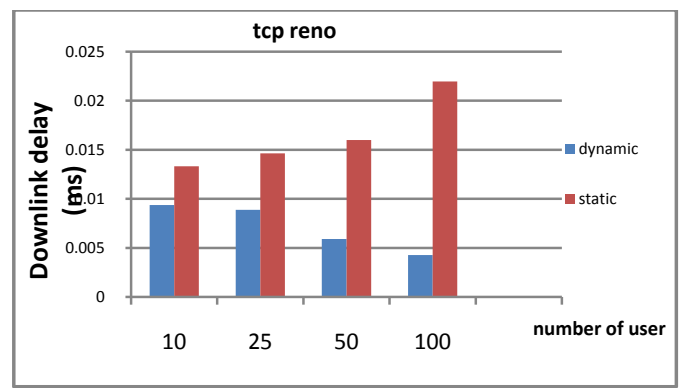

Fig 16. Delay with Reno

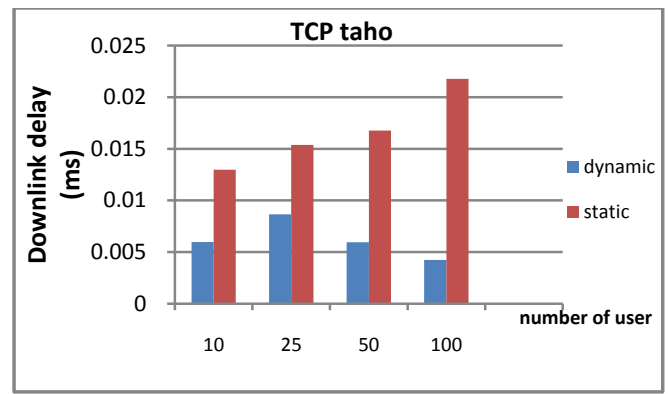

Fig1. Delay with Taho

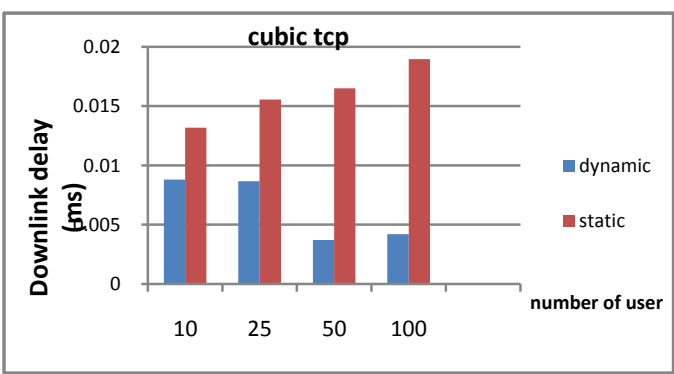

Fig 2. Delay with cubic

\section{CONCLUSIONS}

In LTE Network Several TCP variants have been proposed. It used to enhance quality of service $(\mathrm{QoS})$. In this paper a Enhance approach is presented to avoid buffer overflow at eNode B through controlling the Receive Buffer (bytes) and Maximum Segment Size (bytes) at side in users. The proposed approach made a dynamic change of window size of TCP flow according to the maximum buffer size and the number of users in the cell. For any new TCP session. The simulation result shown efficiency on system performance improvement in terms of network throughput, low delay. 


\section{REFERENCES}

[1] L., K. Sandrasegaran, H. A. M. Ramli, and R. Basukala, "Optimized performance evaluation of LTE hard handover algorithm with average RSRP constraint,", International Journal of Wireless \& Mobile Networks (IJWMN) Vol. 3, No. 2, April 2011.

[2] 3GPP TR 25.913 V9.0.0, "Requirements for Evolved UTRA (E-UTRA) and Evolved UTRAN (E-UTRAN) (Release 9)," Sophia Antipolis Valbonne, France: technical specification group radio access network, the 3rd Generation Partnership Project, Dec. 2009.

[3] M. Mohammed, M. El Bekkali, S. Mazer, M. El Ghazi, and A. Najid, "LTE network capacity analysis to avoid congestion for real time traffic," , IEEE In Proceedings Mediterranean Microwave Symposium (MMS), pp. 1-5. 2014.

[4] K. Jamshaida, B. Shihadaa , A. Showaila , P. Levis, "Deflating link buffers in a wireless meshnetwork," , 16:266 Journal Ad Hoc Networks, Elsevier, pp266-280, 2014.

[5] P. G. Vanparia, Y. R. Ghodasara, "Comparing Study of Transport Layer Protocols SCTP with TCP and UDP,", International Journal of Advanced and Innovative Research (2278-7844) // Vol. 3, no 223 Issue 12. 2014

[6] D. Lisa, Rosario G. Garroppo, S. Giordano, G. Procissi, S. Roma, G. Foddis, and S. Topazzi. , "Advances in lte network monitoring: A step towards an sdn solution,",17 th IEEE Mediterranean Electro technical Conference, pp. 339-343, In MELECON 2014.

[7] P. Bhat, S. N. Campoy, L. Campoy , I. Berberana, T. Derham, G. Liu ,X. Shen, P. Zong , J. Yang ,"LTEadvanced: anoperator perspective ,"IEEE Communications Magazine, vol. 50, no 2, pp.104-114. 2012.

[8] http://www.bectechnolgies.net/maim/newaletter_images. QoS.pdf,1/Aug./2016.

[9] A. A. Atayero, M. K. Luka, M. K. Orya, J.t O. Iruemi, “ 3GPP long term evolution: Architecture, protocols and interfaces," ,International Journal of Information and Communication Technology Research,Vol. 1 no. 7, pp.306-310, November 2011.

[10] G. holland , N. vaidya, "Analysis of TCP performance over mobile ad hoc networks," , Kluwer Academic Publishers. Manufactured in The Netherlands Wireless Networks, vol. 8,no 2,issu 3, pp.275-288,2002.
[11] A. Ghassan, M.Ismail, and K. Jumari, "A survey on performance of congestion control mechanisms for standard TCP versions," ,Journal of Basic and Applied Sciences 5 Australian, vol. 5, no 12,pp. 1345-1352. 12 (2011).

[12] K. I. Oyeyinka1, A. O. Oluwatope, A. T. Akinwale, O. Folorunso, G. A. Aderounmu, O.O. Abiona ,"TCP Window Based Congestion Control-Slow-Start aproach,", Published Online Communications and Network,vol. 3, no 02, pp.85. May 2011.

[13] S. Jain and G.Raina, "An experimental evaluation of CUBIC TCP in a small buffer regime,", IEEE Communications (NCC), National Conference pp. 1-5. 2011.

[14] B., S., L. Sassatelli, D. Collange, D. Lopez-Pacheco, and G. Urvoy-Keller, "Understanding TCP cubic performance in the cloud: A mean-field approach,", IEEE 2nd International Conference In Cloud Networking (Cloud Net), , pp. 190-194. 2013.

[15] L. Zhao, L. Ping, chumming Wu, Q. Yang ,"Avoiding the Evolved Node B Buffer Overflow by using Advertisement Window Control,” ,IEEE Communications and Information Technologies (ISCIT) pp. 268-273. 2011

[16] F. Fatima, B. Doan ,Hoang, "LTE_FICC: A New Mechanism for Provision of $\mathrm{QoS}$ and Congestion Control in LTE/LTE-Advanced Networks, ”, Springer International Publishing, In International Conference on Mobile and Ubiquitous Systems ,Computing, Networking, and Services, pp. 768-781. 2013.

[17] H. Lee , K. Han, “ Buffer overflow notification protocol at link level for wireless ad hoc networks," ,IEEE ELECTRONICS LETTERS Vol. 36 ,No. 22 pp.18991900. 26 th October 2000

[18] K. Jamshaid , B. Shihada , L.Xia, and P. Levis, "Buffer sizing in 802.11 wireless mesh networks, "IEEE Eighth International Conference on Mobile Ad-Hoc and Sensor Systems, pp. 272-281, 2011

[19] A. Ghassan, M.Ismail, and K. Jumari, “ Modeling and performance evaluation of LTE networks with different TCP variants,", International Journal of Electrical, Computer, Energetic, Electronic and Communication Engineering, Vol. 5, No .3, 5, pp.1121-1124. 2011. 